\title{
Apoyo social familiar para el bienestar de hijos gays e hijas lesbianas
}

Linda Teresa Orcasita, Mg.

Pontificia Universidad Javeriana Cali, Colombia*

Teresita María Sevilla, Ph.D.

Pontificia Universidad Javeriana Cali, Colombia**

Victoria Eugenia Acevedo-Velasco, Psy.D.

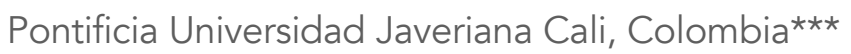

\author{
José Luis Montenegro

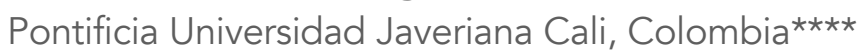 \\ María Camila Tamayo \\ Pontificia Universidad Javeriana Cali, Colombia ${ }^{\star \star \star \star \star}$ \\ Juan Sebastián Rueda-Toro

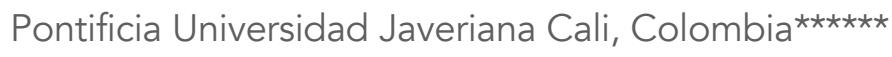

Itorcasita@javerianacali.edu.co

\section{Resumen (analítico)}

Este artículo presenta una revisión sobre los procesos de revelación y aceptación de la orientación sexual y el papel del apoyo social familiar como un aspecto fundamental para el bienestar de hijos gays e hijas lesbianas. Se resalta que los padres, madres, hijos e hijas atraviesan diferentes etapas para lograr la aceptación de la orientación homosexual. Así mismo, el apoyo informacional permite el apoyo emocional por parte de los padres y madres. Se evidencia que ciertas creencias, estereotipos e información insuficiente, así como la pobre educación acerca de la orientación homosexual obstaculizan el apoyo social brindado por las familias a sus hijos e hijas. Se recomienda para futuras investigaciones el abordaje del apoyo social familiar, entendido como un proceso transversal, presente y activo en las familias con hijos gays e hijas lesbianas.

\section{Palabras clave}

Apoyo social, familia, orientación sexual.

\section{Thesauro}

Descriptores en ciencias de la salud.

\section{Para citar este artículo}

Orcasita, L. T., Sevilla, T. M., Acevedo-Velasco, V. E., Montenegro, J. L., Tamayo, M. C., \& Rueda-Toro, J. S. (2019). Apoyo social familiar para el bienestar de hijos gays e hijas lesbianas. Revista Latinoamericana de Ciencias Sociales, Niñez y Juventud, 18(2), 1 23. http://dx.doi.org/10.11600/1692715x.18205

\section{Historial}

Recibido: 17.05.2019

Aceptado: 05.09.2019

Publicado: 18.05.2020

\section{Información artículo}

El artículo forma parte de la revisión de investigaciones para el proyecto con perspectiva cualitativa «Dinámicas familiares frente al proceso de revelación y aceptación de la orientación sexual de hijos gays e hijas lesbianas de la ciudad de Cali-Colombia» (Código 020100594) (Convocatoria capital semiIla), realizado entre el 1 de febrero y el 14 de diciembre de 2018. Financiado por la Oficina de Investigación, Desarrollo e Innovación de la Pontificia Universidad Javeriana Cali. Gran área de conocimiento: Ciencias sociales; Área: Psicología; Subárea: Psicología. 


\section{Family social support for the wellbeing of their gay and lesbian children}

\section{Abstract (analytical)}

This article presents a thematic review of the processes of coming out and acceptance of the sexual orientation of lesbian and gay children and the fundamental role of social support for their welfare. It highlights that parents and sons/daughters go through different stages to achieve acceptance of homosexual orientation. In addition, the study finds that information facilitates emotional support from parents. It is evident that certain beliefs, stereotypes, and insufficient and false information, as well as limited education about homosexual orientation, act as obstacles for providing social support to lesbian and gay children by their families. Recommendations for further research include considering family social support as a crosscutting process that is present and active in families with gay and lesbian children.

Keywords

Social support, family, sexual orientation.

\section{Apoio social familiar para o bem-estar de crianças lésbicas e gays}

Resumo (analítico)

O artigo apresenta uma revisão temática do processo de revelação e aceitação da orientação sexual e o papel fundamental que o apoio social desempenha para o bem-estar de crianças lésbicas e gays. Destaca-se que pais e filhos/filhas passam por estágios diferentes para chegar à aceitação da homossexualidade. Além disso, apoio informacional permite apoio emocional por pais e mães. É evidente que algumas crenças, esteriótipos e informações falsas e insuficientes, assim como baixo nível de educação sobre a homossexualidade se tornam obstáculos para prover apoio social para filhas e filhos lésbicas e gays por parte de suas famílias. Recomenda-se para pesquisas futuras, a abordagem de apoio social familiar, entendendo que esse é um processo transversal presente e ativo nas famílias com filhos e filhas gays e lésbicas.

\section{Palavras-chave}

Apoio social, família, orientação sexual.

\section{Información autores}

[*] Psicóloga y magíster en Familia de la Pontificia Universidad Javeriana Cali. Profesora del Departamento de Ciencias Sociales. Directora e Investigadora del Grupo Bienestar, Trabajo, Cultura y Sociedad (Categoría A1 Colciencias) de la Pontificia Universidad Javeriana Cali. Orcid: 0000-0002-7599-9280. H5: 12. Correo electrónico: Itorcasita@javerianacali.edu.co

[**] Socióloga de la Universidad del Valle y doctora en Sociología de Queen's University of Belfast. Decana de la Facultad de Humanidades y Ciencias Sociales, profesora del Departamento de Ciencias Sociales e investigadora del Grupo Bienestar, Trabajo, Cultura y Sociedad (Categoría A1 Colciencias) de la Pontificia Universidad Javeriana Cali. Orcid 0000-0002-0366-908X. H5: 5. Correo electrónico:

tsevilla@javerianacali.edu.co

$\left.{ }^{[\star * \star}\right]$ Psicóloga de la Universidad de los Andes, magíster en Couseling Psychology de la Tufts University y doctora en Psicología en Massachusetts School of Professional Psychology. Profesora titular del Departamento de Ciencias Sociales e investigadora del Grupo Bienestar, Trabajo, Cultura y Sociedad (Categoría A1 Colciencias) de la Pontificia Universidad Javeriana Cali. Orcid: 0000-0002-1656-7293. H5: 6. Correo electrónico: veacevedo@javerianacali.edu.co

[****] Psicólogo de la Pontificia Universidad Javeriana Cali. Joven investigador Colciencias e investigador del Grupo Bienestar, Trabajo, Cultura y Sociedad (Categoría A1 Colciencias) de la Pontificia Universidad Javeriana Cali. Orcid: 0000-0001-9360-8338. H5: 1. Correo electrónico: joseluis.montenegro@javerianacali.edu.co

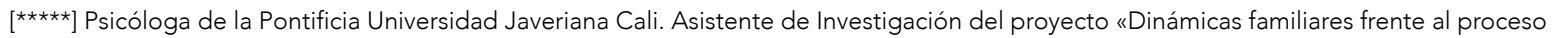
de revelación y aceptación de la orientación sexual de hijos gays e hijas lesbianas de la ciudad de Cali-Colombia», realizado por el Grupo Bienestar, Trabajo, Cultura y Sociedad (Categoría A1 Colciencias) de la Pontificia Universidad Javeriana Cali, en 2018. Orcid: 0000-0002-9517-8930. H5: 0. Correo electrónico: mariac.tamay094@gmail.com

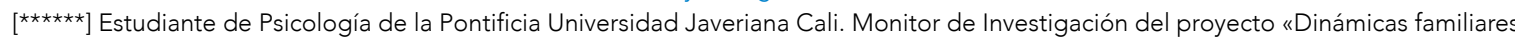
frente al proceso de revelación y aceptación de la orientación sexual de hijos gays e hijas lesbianas de la ciudad de Cali-Colombia», realizado por el Grupo Bienestar, Trabajo, Cultura y Sociedad (Categoría A1 Colciencias) de la Pontificia Universidad Javeriana Cali, en 2018. Orcid: 0000-0003-3824-4310. H5: 0. Correo electrónico: sebastianrueda@javerianacali.edu.co 


\section{Introducción}

$\mathrm{C}_{\text {social de las personas, en tanto que favorece el crecimiento desde lo individual }}$ hasta los vínculos que se establecen con los diferentes agentes de socialización, incluso permitiendo sobrellevar situaciones de adversidad. Feeney y Collins (2015) mencionan que cuando las relaciones humanas son nutritivas, el apoyo social fortalece el crecimiento personal en las diferentes etapas del desarrollo y brinda herramientas para afrontar situaciones percibidas como negativas y estresantes. Orcasita y Uribe (2010), a su vez, en su revisión sobre aspectos fundamentales del apoyo social para el bienestar en la adolescencia, encuentran que este comprende tres tipos de apoyo: emocional, material e informacional. El primero se refiere a la expresión de sentimientos, preocupaciones, actividades de cuidado e intimidad; el segundo es la forma de ofrecer herramientas o servicios frente a situaciones concretas; mientras que el tercero hace alusión a brindar algún tipo de información con el propósito de aconsejar $\mathrm{u}$ orientar a la otra persona.

Diversas investigaciones internacionales han encontrado que el apoyo social que brindan las familias favorece el proceso de desarrollo integral de las personas, especialmente en aquellas que se reconocen con orientaciones sexuales diversas (lesbiana, gay, bisexual _LGB_) (Jiménez \& Romero, 2014; Kabakov, 2014; Lagazzi, 2016; Mustanski, Newcomb \& Garofalo, 2011; Snapp, et al., 2015; Svab, 2016). En cuanto a la investigación colombiana en este tema, se encuentra que esta es incipiente, pues los esfuerzos se han enfocado en investigar el desarrollo de la identidad homosexual y las experiencias de familias frente a la revelación desde sus representaciones de ser hombre o mujer en sociedad (Montenegro et al., 2019; Rueda, 2015; Vargas et al., 2011; Zambrano et al., 2017; Zambrano et al., 2019).

De esta manera, poco se ha documentado sobre el apoyo familiar y el papel que este juega en el proceso de revelación de la orientación sexual de hijos gays e hijas lesbianas en familias colombianas. Así que deberían identificarse recursos de los individuos y de 
sus relaciones, así como estrategias útiles empleadas por las familias que facilitan movilizaciones en el tiempo a nivel de la comunicación, la expresión de los afectos, la construcción de nuevos significados y formas alternativas para abordar las situaciones difíciles. Una mirada más focalizada sobre el apoyo social percibido, que emerge desde las experiencias de estas familias y otros sistemas más amplios (en formas dinámicas y complejas) permitiría identificar con mayor claridad cuáles y de qué manera los diferentes tipos de apoyo social contribuyen al desarrollo de procesos familiares (organizativos, comunicacionales, afectivos, de construcción de significado), favoreciendo así el bienestar para quienes develan su orientación sexual y el de todos los involucrados.

En Colombia dos encuestas permiten vislumbrar la posición de las familias frente a sus hijos gays e hijas lesbianas: la primera es la Encuesta Nacional de Demografía y Salud (Ministerio de Salud y Profamilia, 2015), la cual encontró que, aproximadamente, el 6o \% de las mujeres y el $72 \%$ de los hombres participantes no apoyaría un hijo gay o a una hija lesbiana. La segunda encuesta desarrollada por las organizaciones Colombia Diversa y Sentiido (2016) sobre el clima escolar en 581 adolescentes y jóvenes LGBT (lesbianas, gays, bisexuales y transexuales), reportó que el 70.2\% ha sufrido alguna vez de acoso verbal, el $47.3 \%$, acoso sexual y el 15.9\%, agresión física por su orientación sexual. Únicamente el $39.9 \%$ informó a algún familiar sobre estas situaciones y, de ese segmento, solo el 73.5\% realizó alguna intervención posterior de seguimiento.

Es posible pensar que las familias cuentan con pocos recursos, estrategias o herramientas de acompañamiento a hijos gays e hijas lesbianas, dado que predominan y coexisten discursos sociales, religiosos, muchos de ellos homofóbicos y heteronormativos y ya que falta información social con respecto a la homosexualidad (Svab, 2016). Lo anterior se puede traducir en dificultades para brindar apoyo y, a su vez, promover un adecuado ajuste y bienestar físico, psicológico y familiar. Igualmente, diversos estudios han señalado que los procesos de revelación de la orientación homosexual en el contexto familiar por algún hijo o hija están asociados a diversas situaciones o elementos que pueden facilitar o retrasar un ajuste saludable/pertinente. En estos se encuentran la edad del hijo o hija al momento de la revelación, la presencia/ausencia de redes de apoyo, las creencias, prejuicios y conocimientos, así como la intuición de la orientación homosexual por parte de los padres y madres (Pérez, 2005; Phillips \& Ancis, 2008; Quintanilla et al., 2015; Savin-Williams \& Dubé, 1998; Solís, 2014).

Como parte de estos procesos, se reconoce también que existen escenarios posteriores a la revelación que pueden estar asociados a los factores señalados. Svab (2016) pro- 
pone la noción de un estado denominado «el closet transparente» para referirse al momento posterior a la revelación en el que prevalece el silencio familiar frente a la orientación sexual del hijo/a (recientemente compartida); silencio que puede ser interrumpido por momentos de chantaje emocional y conflictos, lo que permite que parezca que nada ha cambiado al interior de la familia, generando tensión en los distintos miembros del sistema debido a la imposibilidad de expresar lo que piensan y sienten al respecto.

Desde un enfoque propositivo, estos procesos permiten transformar concepciones personales y familiares en los miembros. Diversas investigaciones coinciden en afirmar que dichos procesos fortalecen los vínculos relacionales, generan mayor comunicación, expresión de afecto y cuidado, disminución de niveles de homofobia internalizada, mayores niveles de apoyo familiar, así como transformaciones en las creencias sobre las orientaciones sexuales no hegemónicas al interior de las familias (González et al., 2013; Luján \& Tamarit, 2012; Orcasita et al., 2015; Pérez, 2005). Desde una perspectiva social, la familia de origen juega un papel significativo en que el hijo gay o la hija lesbiana desarrollen recursos para considerar, planear y tomar acciones que les permitan reclamar sus derechos como ciudadanos/as no heterosexuales (Chan, 2017).

Ahora bien, en la presente revisión se parte de las siguientes concepciones de familia y diversidad sexual. La familia se concibe como una organización primaria e interdependiente de una o más personas, ya sea por lazos de consanguinidad, vínculo afectivo o parentalidad no consanguínea, que procura brindar bienestar a quienes la conforman. Tiene un rol como agente socializador para desarrollar al ser humano, educarlo e integrarlo a su contexto sociocultural. Se trata pues de modelos jerárquicos y con microsistemas (conyugal, parental y fraternal) que facilitan el desarrollo individual y colectivo por medio de la satisfacción de necesidades psicológicas, culturales y biológicas. Así mismo, se construyen unas reglas que median la interacción interna y externa, las cuales facilitan la construcción de una identidad en niveles individuales y colectivos desde lo familiar (Espinal et al., 2006; Hernández, 2001).

De igual manera, las familias inculcan valores, actitudes y prácticas sociales que tienden a conformarse con los roles de género, tradicionalmente asociados al sexo y los cuales, a su vez, van a influir en la expresión de feminidades y masculinidades entre hombres y mujeres, asociadas por algunas familias con la heterosexualidad (Hernández, 2006). No obstante, las familias se pueden reconfigurar y transformar a partir de pensamientos, sentimientos o acciones de uno de sus integrantes, así como sucede cuando un hijo o hija revela a su familia que se identifica con una orientación homosexual. Inicialmente, ello 
puede dar paso a reacciones negativas y, seguidamente, a un proceso de deconstrucción y resignificación de creencias, valores, comportamientos, conceptos e imaginarios en relación a la familia, la sexualidad y a lo que es ser hombre y mujer en la sociedad (CeballosFernández, 2014; Saltzburg, 2007).

En cuanto a la diversidad sexual, según Núñez (2016), esta comprende y abarca de manera integral expresiones, experiencias, vivencias, emociones e identidades vinculadas con la sexualidad, sin distinguir raza, género, orientación sexual o sexo, entre otras. En este sentido, debe incluir la heterosexualidad. Sin embargo, en las sociedades occidentales coexisten diferentes discursos médicos, religiosos, morales o sociales que armonizan o conflictúan la vivencia de la sexualidad y, por lo tanto, la diversidad sexual (Szasz, 2004).

Dado lo anterior, la presente revisión busca reflexionar sobre el papel que juega el apoyo social familiar en los procesos de revelación y aceptación de la orientación sexual de hijos gays e hijas lesbianas pues, como se ha expresado, a nivel nacional es poca la literatura científica que existe sobre el tema. Ello permitirá seguir avanzando en estos estudios, teniendo en cuenta las necesidades apremiantes para apoyar a las familias colombianas.

\section{Método}

La búsqueda de las investigaciones se realizó entre abril de 2017 y enero de 2019, a través de las bases de datos ProQuest, Ebsco, Scopus, NCBI, SciELO, Taylor \& Francis y Google Académico, por medio de las palabras claves: «procesos de revelación», «familia», «orientación sexual», «hijos gays», «hijas lesbianas», «homosexual» $\mathrm{y}$ «apoyo social» (tanto en inglés como en español). Posteriormente, se seleccionaron las investigaciones que cumplieran con los siguientes criterios: a) indagación en cómo fueron los procesos de revelación de hijos gays e hijas lesbianas a padres y madres; b) las reacciones en los contextos familiares frente a la orientación sexual diversa; c) apoyo social que percibieron hijos gays e hijas lesbianas por parte de sus contextos de socialización (familia, amigos, colegios); d) que hubiesen participado jóvenes gays, lesbianas, bisexuales, padres y madres en dichas investigaciones; y e) investigaciones publicadas entre 2008 y 2019.

A partir de ello, fueron identificadas 29 investigaciones a nivel internacional, siete a nivel latinoamericano y seis a nivel nacional, a las que se les realizaron reseñas en búsqueda de diferencias y similitudes en sus resultados y conclusiones. Especialmente se incluyen autores que son reconocidos internacionalmente y a nivel nacional por el aborda- 
je de familias y diversidad sexual y de género, centrada en los últimos 11 años en su mayoría de Norteamérica y Latinoamérica, seguido por Europa y Asia. Adicionalmente, se incluyeron documentos para la contextualización y la definición de términos que fundamentan la problematización y los aspectos teóricos del artículo sobre revisión del tema enfocados epistemológicamente en la compresión de la diversidad sexual y de género en las familias desde una perspectiva construccionista, en la cual los escenarios de socialización permean los imaginarios sociales en las familias.

\section{Resultados}

\section{Procesos de revelación de hijos gays e hijas lesbianas}

Los procesos de revelación de hijos gays e hijas lesbianas pueden incluir diversas etapas o fases con variaciones en estas, según la experiencia de cada hijo o hija con su orientación sexual, como han podido establecer investigaciones a nivel nacional e internacional (Aranda et al., 2015; Guittar \& Rayburn, 2016; Pistella et al., 2016; Tamagawa, 2018; Vargas et al., 2011; Zambrano et al., 2019).

Diversos estudios han encontrado que las edades de la revelación en los contextos familiares y sociales por parte de un hijo gay o una hija lesbiana, de manera general, se ubican entre los 10 y 25 años (Apoorva \& Thomas, 2016; Baiocco et al., 2015; Ryan et al., 2015), aunque algunos estudios reportan que los hijos e hijas homosexuales reconocen su atracción hacia su mismo sexo en la infancia (Jiménez \& Romero, 2014; Tyler, 2015). Adicionalmente, Aranda et al. (2015) encontraron que características étnico-raciales podrían influir en la edad de revelación; en su estudio cuantitativo en Norteamérica (con 351 mujeres lesbianas), las participantes afroamericanas divulgaron su orientación a los 22.77 años, las latinas a los 23.24 y las blancas a los 25.23. No obstante, autores como Guittar y Rayburn (2016) plantean que la revelación de la orientación sexual de personas LGB no debería ser vista como un proceso delimitado con un inicio y finalización reconocibles, sino como una trayectoria sin fin, pues exige una gestión interna y externa constante de una identidad que resulta marginal para la sociedad.

Previo a la revelación familiar y social de la orientación sexual no hegemónica por parte de hijos e hijas, ellos y ellas atraviesan momentos de relevación y aceptación de su orientación consigo mismos. Figueroa y Tasker (2013), Jiménez y Romero (2014), Quintanilla et al. (2015) y Ocampo (2014), en investigaciones cualitativas desarrolladas en Mé- 
xico, Estados Unidos y Chile con personas homosexuales, coinciden en señalar que la construcción de la identidad homosexual atraviesa diferentes etapas, como el descubrimiento, la confusión, la conciencia, la aceptación y la integración que, a su vez, concuerda con lo planteado, en su momento, por Vivian Cass (1979) en la década de los setenta. Dichas etapas se caracterizan por que los hijos gays y las hijas lesbianas descubren que les gustan las personas de su mismo sexo, experimentan encuentros íntimos con amigos o compañeros, lo cual genera confusión, pues no es lo socialmente establecido y, al mismo tiempo, prefieren ocultarla a su familia o personas de su entorno. Adicionalmente, Bond, Hefner y Drogos (2009) describen la significativa influencia que medios como el internet tienen en el desarrollo de la identidad sexual de individuos LGB, afirmando que, a través de la búsqueda de información, comunicación con pares con su misma orientación sexual e incluso confirmación y realización de sus deseos a través de contenidos sexuales, logran aceptar e integrar su orientación sexual a sus identidades.

Luego se da inicio al proceso de aceptación de la orientación homosexual —lo cual se puede facilitar por la aceptación en el contexto familiar-y favorece el bienestar físico y psicológico. Igualmente, el rechazo por parte del núcleo familiar afecta de forma negativa dimensiones personales y sociales que obstaculizan el ajuste e integración de la orientación sexual diversa. Por último, se genera la integración de esta orientación como un aspecto y no una totalidad de la persona, permitiendo su sincronización de manera armónica con otros aspectos individuales, familiares y sociales (Figueroa \& Tasker, 2013; Jiménez \& Romero, 2014; Ocampo, 2014; Quintanilla et al., 2015).

Baiocco et al. (2015) y Mustanski et al. (2011), en sus estudios cuantitativos realizados en Estados Unidos e Italia, hallaron que los jóvenes homosexuales participantes tenían más probabilidades de divulgar su orientación sexual a un amigo o hermanos primero que a sus padres, en coherencia con lo encontrado por Perrin-Wallqvist y Lindblom (2015) en un estudio similar en Suecia. Tales procesos podrían presentar variaciones asociadas a la etnia y el sexo, pues los latinos, afrodescendientes y mujeres reportaron menos posibilidades de contarle a sus padres (Mustanski et al., 2011).

Por su parte, Baiocco et al. (2015) hallaron correlaciones significativas en la forma en que los padres y madres se dieron cuenta de la orientación sexual de sus hijos o hijas: las madres tuvieron mayores probabilidades de que sus hijos o hijas les contaran sobre ello (65.2\%) o ellas de preguntarles directamente (9.8\%); en comparación con los padres, quienes tuvieron más probabilidades de darse cuenta por sí mismos (32.3\%). Las madres pueden conocer de la orientación homosexual de su hijo o hija antes que los demás miem- 
bros, tal como lo encontraron Trussell, Xing y Oswald (2015) en una investigación cualitativa con padres y jóvenes LGB, donde ellas se consideraron como red de apoyo relevante para sus hijos e hijas, así como agentes reconciliadoras en las relaciones intrafamiliares respecto a la orientación homosexual. No obstante, es importante reconocer matices asociados a esta relación entre el género de los hijos e hijas, el género los padres y las madres y la revelación. Como se observa en la investigación realizada en Japón por Tamagawa (2018) con 43 participantes y que coincide con los hallazgos resultantes de estudios occidentales, los participantes consideraron más difícil revelar su orientación sexual a sus padres que a sus madres, pero las respuestas de las madres fueron generalmente reactivas y de abuso, especialmente para las mujeres lesbianas y mujeres bisexuales, mientras que frente a los hombres gays las madres fueron menos demostrativas.

Adicionalmente, Pistella et al. (2016) afirman que el alto nivel educativo y las posiciones políticas familiares liberales son también factores que facilitan la revelación, pues se relacionan con reacciones positivas por parte de sus padres y madres. Además, los hijos gays e hijas lesbianas que tienen más probabilidades de revelar su orientación sexual no hegemónica en sus contextos familiares son quienes cuentan con amplias redes de apoyo, especialmente con personas que se autodenominan de su misma orientación sexual y están en relaciones de pareja (Aranda et al., 2015; Pistella et al., 2016). No obstante, Ronzón-Tirado et al. (2017) buscando describir los elementos relacionados con los modelos parentales y la violencia ejercida en parejas gays y lesbianas, encontraron que tanto las dinámicas de violencia entre los padres como el rechazo de su orientación sexual no hegemónica al momento de la revelación, se convierten a futuro en factores que dificultan la capacidad de estas personas para rastrear y enfrentar la violencia en sus propias relaciones de pareja.

En un estudio cualitativo con 130 jóvenes LGB, Manning (2015) estableció unas categorías de conversaciones sobre cómo estos participantes revelaron su orientación sexual a otras personas, entre las que se incluyen: planificadas, emergentes, enjauladas, de confrontación, románticas y sexuales y mediadas. Estas conversaciones se diferencian a partir de la decisión propia, la presión social o las eventualidades del contexto que generaron que estos participantes divulgaran, de una forma u otra, su orientación homosexual a otros. Las conversaciones planificadas brindan mayores posibilidades, ya que se elabora un discurso para dar apertura a la revelación y se realizan por voluntad propia.

Dichos procesos de revelación por parte de hijos gays e hijas lesbianas, de igual manera, se encuentran atravesados por sentimientos en las diferentes etapas o fases, como 
lo encontraron Perrin-Wallqvist y Lindblom (2015) en un estudio cualitativo en Suecia con seis participantes gays y lesbianas. Estos sentimientos fueron de alienación, malestar y miedo, así como de autoaceptación, comodidad con la sexualidad propia y un sentimiento de completitud. En primera instancia, los participantes manifestaron haber vivido procesos de negación de su atracción hacia personas de su mismo sexo, como reacción a transgredir lo socialmente establecido, lo cual les llevó también a generar sentimientos de malestar y miedo por las reacciones de sus figuras parentales. En segundo lugar, otros expresaron que las relaciones con personas de su mismo sexo les permitieron confirmar su orientación sexual y favorecer la integración y el ajuste de esta a sus identidades.

Por otra parte, según estudios cualitativos realizados en diferentes partes del mundo (Estados Unidos, India, México, Suecia y Chile) con jóvenes gays y lesbianas encontraron que sienten confusión por el gusto hacia personas de su mismo sexo, lo cual se puede afrontar, desde la negación y la homofobia, hasta con la naturalidad de su orientación sexual diversa. Ello se percibe como una forma de evitar reconocerse, ocultarla para equilibrar entre su atracción y lo socialmente establecido y preguntarse sobre el origen. Algunos estudios han hallado reacciones que pueden describirse como negativas en jóvenes gays y lesbianas. Estas se asocian a la vergüenza, malestar, miedo, temor y tristeza, lo que incluso puede generar ideación o conductas suicidas (Quintanilla et al., 2015). Por último, cuando atraviesan el proceso del reconocimiento personal y familiar, las reacciones que se generan son de libertad, orgullo, alivio y aceptación de sí mismos (Apoorva \& Thomas, 2016; Biswas \& Chaudhuri, 2018; Figueroa \& Tasker, 2013; Jiménez \& Romero, 2014; Perrin-Wallqvist \& Lindblom, 2015; Tyler, 2015).

\section{Procesos de aceptación de padres y madres con hijos gays e hijas lesbianas}

Para los padres y madres el conocer la orientación sexual no hegemónica de su hijo o hija puede ser difícil, dadas las creencias arraigadas social e históricamente sobre los modelos «naturales» de familia, los roles de género de sus miembros y la orientación heterosexual. De ahí que la homosexualidad emerja como una construcción transgresora de dichas creencias y expectativas que tienden a ser reproducidas de una generación a otra (Bautista, 2018). Revisiones empíricas al respecto señalan que, por ejemplo, en México el concepto de familia se basa en la masculinidad y la heterosexualidad (realidad no ajena a la sociedad colombiana), lo que hace que el proceso de aceptación de la homosexualidad por parte de los padres, especialmente de los hijos hombres, sea más difícil y no se reciba 
apoyo y aceptación inmediata (Quintanilla et al., 2015). Este aspecto difiere más en el caso de las mujeres lesbianas, pues socialmente se validan expresiones de afecto, intimidad y cercanía entre ellas.

Así como los hijos e hijas homosexuales viven su proceso de revelación y aceptación de la orientación sexual consigo mismos y sus contextos familiares, los padres y las madres también atraviesan por un proceso de revelación a sí mismos que, posteriormente, se amplía a otros entornos sociales. Las figuras parentales pueden atravesar todas, algunas o ninguna de las etapas sin un orden establecido, así como saltarse o estancarse en una etapa (Kabakov, 2014; Savin-Williams \& Dubé, 1998).

De acuerdo con Savin-Williams y Dubé (1998), los padres y madres experimentan cinco etapas desde el momento en que su hijo o hija divulga su orientación homosexual en el contexto familiar, similares a las que se identifican en un proceso de duelo: negación y aislamiento (que podría ser asimilada también a la etapa de «closet transparente» mencionada por Svab, 2016); ira; negociación; depresión; y, por último, aceptación. Estas también se asemejan a lo que han encontrado o planteado por investigaciones posteriores (Baptist \& Allen, 2008; Cassar \& Grima, 2016; Tyler, 2015; Uribe et al., 2018).

Un primer momento se acompaña con una reacción de shock de manera inmediata. No obstante, tal reacción no siempre se da de manera marcada, debido a que las figuras parentales han podido intuir o sospechar de la orientación sexual de su hijo o hija previamente, a través de conversaciones telefónicas, amistades, relaciones de pareja, entre otros (Savin-Williams \& Dubé, 1998).

Savin-Williams y Dubé (1998) también mencionan que los padres y madres afrontan la noticia —en esa primera etapa - desde la negación: consideran que es una fase de experimentación de los hijos e hijas y optan por alejarse familiar y socialmente. Luego pueden atravesar por una etapa de la ira, para disminuir la culpa, la cual puede hacerse evidente por medio de agresiones físicas y verbales o al culpabilizar a personas externas (amigos, vecinos, profesores). Cuando la ira ha disminuido, se da cabida a la negociación, fase en donde los padres y madres acuden a consejeros religiosos o psicoterapeutas para tratar de cambiar o «curar» la orientación sexual no hegemónica, así como persuadir a sus hijos o hijas de que su orientación deber ser un secreto familiar. En esta etapa las figuras parentales pueden ingresar a un momento de depresión por no haber identificado o reconocido la orientación homosexual de su hijo o hija y brindar algún tipo de solución; así como la frustración de que la revelación de la orientación haya sido a agentes externos al núcleo familiar primario. Sin embargo, comienzan a preguntarse sobre la vejez de 
su hijo o hija, o si van a vivir discriminación, lo cual favorece el reconocimiento de la orientación homosexual y, a su vez, les lleva hacia la última etapa. Así pues, la aceptación se facilita a partir de material educativo, grupos de apoyo y su promoción. Dicha etapa se comienza a visibilizar cuando los progenitores no se incomodan por contarles a personas externas sobre los gustos sexuales de sus hijos o hijas.

Cuando se genera el proceso de aceptación este influye en el ámbito político, debido a que los integrantes de la familia pueden construir posturas críticas respecto a los discursos hegemónicos y otros aspectos que pueden afectar negativamente el desarrollo personal y social de las personas homosexuales (Baptist \& Allen, 2008).

Adicionalmente, estas etapas se encuentran permeadas por reacciones, sentimientos, creencias y pensamientos de los padres y madres cuando se enteran de la orientación homosexual de sus hijos(as). Varias investigaciones (Cassar \& Grima, 2016; Goodrich, 2009; Lagazzi, 2016; Luján \& Tamarit, 2012; Phillips \& Ancis, 2008; Pinos et al., 2011; Solís, 2014) han encontrado que los padres y madres tienen reacciones negativas y positivas que pueden favorecer u obstaculizar los procesos de aceptación. Las reacciones negativas (las cuales se presentan generalmente en momentos posteriores inmediatos a la revelación explícita) son: miedos, angustia y temores por la discriminación social, transmisión de ITS/VIH, proyectos de vida, evitación o negación del tema, rechazo, ira, castigo físico (que puede ser ejercido por las creencias y prejuicios acerca de las personas homosexuales), esperanza de cambiar la orientación sexual, confusión, vergüenza, autoculpa (por haber fallado en la crianza o culpabilizar a otros por ello), búsqueda de una causa, ocultar a otros la orientación sexual y, por último, sentirse heridos por la falta de confianza de los hijos o hijas (aunque ello depende de la edad de la revelación).

Finalmente, algunas reacciones positivas que manifiestan haber tenido padres y madres después de las reacciones negativas y de lograr normalizar la revelación de la orientación sexual homosexual de su hijo/a fueron: búsqueda profesional o material educativo sobre dicha orientación para comprenderla, tolerancia, conocer a personas con orientaciones sexuales diversas, reestructuración en las creencias construidas sobre la orientación homosexual y religiosas; así como sentimientos de orgullo, valentía y confianza por sus hijos o hijas hasta la aceptación incondicional (Cassar \& Grima, 2016; Goodrich, 2009; Lagazzi, 2016; Luján \& Tamarit, 2012; Phillips \& Ancis, 2008; Pinos et al., 2011; Solís, 2014). 


\section{Apoyo social familiar a hijos gays e hijas lesbianas}

El apoyo social familiar influye positivamente en aspectos personales, familiares y sociales en hijos gays e hijas lesbianas. Según los resultados y conclusiones que plantean Baptist y Allen (2008), Bond et al. (2009), Chan (2017), Jiménez y Romero (2014), Ocampo (2014), Phillips y Ancis (2008), Snapp et al. (2015) y Svab (2016), el apoyo social familiar es fundamental para promover el bienestar físico, psicológico y social de hijos gays e hijas lesbianas frente a su orientación sexual, ya que este promueve la autoaceptación, el posicionamiento personal, familiar y social, el tener una vida saludable, la autoestima, así como facilitar que la revelación sea realizada a otros agentes de socialización e, incluso, para considerar, planear o tomar acciones para reclamar sus derechos ciudadanos como personas no heterosexuales (Kabakov, 2014; Lagazzi, 2016; McConnell et al., 2016; Mustanski et al., 2011). Además, si este apoyo se presenta en la etapa de la adolescencia, va a influir en etapas posteriores del ciclo vital (Snapp et al., 2015).

Otras investigaciones (Hatzenbuehler, 2011; Quintanilla et al., 2015) encontraron que los contextos familiares y sociales positivos facilitan que haya un ajuste adecuado e integral de la identidad sexual por encima de aspectos personales, pues los y las participantes en contextos sociales hostiles y negativos reportaron haber intentado suicidarse alguna vez. En el estudio realizado por McConnell et al. (2016), los y las participantes con poco apoyo social familiar reportaron mayor angustia durante la adolescencia y juventud, en comparación con aquellos que tuvieron apoyo social familiar. Asimismo, concluyeron que el apoyo social puede brindar elementos o prevenir problemáticas en salud mental en personas con orientaciones sexuales o identidades de género diversas.

Por otro lado, Bond et al. (2009) aseguran que dada la fuerte influencia de internet en el desarrollo de la identidad sexual de individuos LGB, aquellos que usan más este medio durante el proceso de revelación tienen menos probabilidad de comunicarse abiertamente con sus familias en un momento posterior. Así, aunque internet sirve como un catalizador que impulsa a las personas LGB a revelar su orientación sexual a sus familias, si su identidad sexual es rechazada, estas volverán a sentirse igual que antes y a preferir canales que proveen una sensación de anonimato y un espacio seguro para la comunicación en línea. Es por ello que se requiere de una buena comunicación familiar que promueva menos homofobia internalizada y mayores niveles percibidos de apoyo familiar.

Algunos estudios coinciden en que el apoyo informacional recibido o buscado por los padres y madres es un aspecto relevante para los procesos de revelación y aceptación de la orientación sexual de hijos gays e hijas lesbianas. Generalmente, dicho apoyo es 
brindado a través de los medios de comunicación, así como por profesionales, grupos de apoyo de padres, madres o familiares y personas con orientaciones sexuales diversas, entre otros. Estos recursos informacionales influyen para que los miembros de la familia puedan comprender la orientación sexual desde un enfoque propositivo y conlleva un apoyo emocional (Baptist \& Allen, 2008; Ocampo, 2014; Phillips \& Ancis, 2008; Solís, 2014; Trussell et al., 2015).

Sin embargo, hay que resaltar que ciertos estereotipos — que asocian la homosexualidad únicamente al espectáculo y la diversión (Ceballos-Fernández, 2014), así como creencias, mitos y la falta de información y educación- obstaculizan el apoyo social brindado por la familia (Baiocco et al., 2015; Ceballos-Fernández, 2014; Luján \& Tamarit, 2012). Ello se ha podido relacionar al consumo de sustancias psicoactivas, trastornos mentales, ideación o conducta suicida y homofobia internalizada (Apoorva \& Thomas, 2016; Flentje, Bacca, \& Cochran, 2015; McConnell et al., 2016; Plöderl et al., 2013; Quintanilla et al., 2015), dado que los hijos gays e hijas lesbianas han manifestado que la familia es una red de apoyo primordial e importante para sus vidas. Por ende, si esta no brinda un apoyo, su bienestar psicológico y físico se afecta negativamente (Aranda et al., 2015; Solomon, McAbee, \& McGee, 2015).

Finalmente, Chan (2017) en una investigación llevada a cabo en China, plantea que -al igual que lo develado por investigaciones occidentales-el apoyo de la familia de origen juega un papel muy importante en el bienestar de los individuos LGB, pues influye en la sensación de seguridad o inseguridad que pueden llegar a tener al momento de estar en pareja. Lo anterior se relaciona también con los hallazgos de Ronzón-Tirado et al. (2017) sobre los modelos de violencia aprendidos de los padres y replicados en las propias relaciones de pareja por personas gays y lesbianas. Además, Chan (2017) asegura que los efectos negativos de la exclusión e indiferencia de la familia no solo afectan la salud mental, sino que también darán forma y crearán barreras sociales futuras para la exigencia de derechos ciudadanos de estas personas. Finaliza asegurando que, debido a la importancia que se le otorga a la familia de origen en esta cultura, las personas buscan integrar su identidad sexual a su relación familiar en lugar de aislarse, buscando evitar destruir las relaciones familiares y el honor como su primera prioridad. 


\section{Discusión}

El apoyo social familiar favorece los procesos de revelación y aceptación de hijos gays e hijas lesbianas pues, como se observa que en las investigaciones revisadas, los miembros de las familias atraviesan por diferentes momentos para integrar la orientación sexual no hegemónica en la identidad personal y familiar. Frente a dicho escenario, las familias cumplen un papel fundamental dado que, cuando los padres y madres aceptan la orientación homosexual, a su vez, favorecen la aceptación propia y se genera una sincronización armónica con otros aspectos personales, familiares, sociales y que puede permear etapas posteriores del ciclo vital.

Las crisis y los desafíos particulares de cada familia (como cuando un hijo gay o hija lesbiana revela su orientación sexual) impactan a todos los miembros del sistema. Por tanto, consideramos que introducir una mirada sistémica, relacional y contextual es fundamental para el entendimiento del apoyo social y de su papel en los procesos de revelación y aceptación. De acuerdo con Walsh (2016), estudiosa de familias bajo estrés y pionera en estudios de resiliencia familiar, se reconoce que las familias utilizan recursos clave para mediar la adaptación (o mal adaptación) de todos los miembros, sus relaciones y de la unidad familiar. Dichos recursos emergen en sistemas de creencias compartidas, en los procesos organizativos (los que conducen a la flexibilidad, conexión entre los miembros, movilización de recursos sociales y económicos para la satisfacción de necesidades), en los procesos comunicacionales (donde se logra claridad en la información, apertura emocional para compartir una amplia gama de emociones, incluidas las dolorosas y positivas) y en los procesos de solución de problemas en colaboración (que muevan hacia la recursividad, toma de decisiones compartida, el aprender de las pérdidas, los tropiezos, los errores cometidos, las caídas, y se trabaje hacia la prevención, la proactividad, etc.).

No obstante, como indican las investigaciones contemporáneas, cuando las familias generan rechazo a sus hijos e hijas y a las situaciones por ellos planteadas, puede afectarse negativamente el desarrollo de aspectos personales, familiares y sociales, no solo de estos miembros particulares, sino de todo el sistema familiar en su conjunto. Este rechazo se puede generar por las creencias, estereotipos y estigmas que se han construido en torno a la orientación homosexual (Ceballos-Fernández, 2014) que prevalecen en muchas sociedades, incluida la colombiana. La noticia del reconocimiento de un hijo gay o una hija lesbiana moviliza a todo el núcleo familiar, pues usualmente no cumple con lo socialmente establecido; por lo cual se van a producir transformaciones en su organización y 
relaciones familiares. Estos ajustes, como lo describen las investigaciones revisadas, también pueden favorecer el crecimiento personal y social de los y las integrantes, hasta los padres y madres, quienes en muchos casos pueden lograr, con apoyo externo o desde el agenciar sus propios recursos relacionales, asumir posiciones políticas en pro de las personas homosexuales.

Por su parte, el apoyo informacional a las familias, sea buscado por ellas mismas o brindado por profesionales e instituciones, puede permitir la apertura hacia el entendimiento más profundo de la diversidad sexual en el contexto familiar y social. Lo anterior podría reflejarse en nuevas experiencias emocionales, de conexión positiva, de unión, de solidaridad, de reconocimiento y validación de experiencias no conocidas, así como de temores y dudas, y que se convierten en formas de apoyo emocional que los padres brindan y reciben en interacción positiva con sus hijos gays e hijas lesbianas.

De igual manera, cuando las y los progenitores tienen o perciben una red de apoyo amplia, que puede ayudarlos a través de la contención y validación de sus emociones, se facilita el proceso de revelación a nivel familiar y social de lo que ocurre con su familia. Por lo cual, contar con redes de apoyo sensibles a los temas de diversidad sexual puede prevenir que los hijos experimenten sentimientos de malestar, inseguridad, incomodidad y homofobia hacia su orientación sexual, así como prácticas que afecten negativamente su bienestar como consumo de sustancias, prácticas sexuales sin protección, entre otras.

Así pues, la investigación nos muestra caminos para explorar y trabajar desde la academia y desde otros sectores de la sociedad a fin de facilitar el acceso a información veraz, la cual amplié el panorama de vida y de las transiciones de las familias cuyos hijos revelan su orientación sexual no heterosexual. Documentar las experiencias y socializarlas en medios académicos y no académicos contribuye a generar nuevas representaciones sociales sobre estas familias de las cuales aún se sabe poco. Desde las contribuciones prácticas de los estudios revisados, se recomienda: 1) potenciar la formación en diversidad sexual y de géneros en las familias; 2) brindar espacios que generen redes de apoyo en las familias con hijos gays e hijas lesbianas; 3 ) generar articulación intersectorial especialmente en salud y educación para brindar acceso y garantía de los derechos sexuales y derechos reproductivos en población con orientaciones sexuales no hegemónicas; 4) cualificar la formación de profesionales de la salud y educación para facilitar el acompañamiento efectivo a nivel del apoyo emocional, material e informacional; y 5) desarrollar iniciativas para brindar protección y seguridad a la población que ha experimentado situaciones de violencias y discriminación. 
Además, cabe anotar que, en los estudios revisados, las madres se convierten en una red de apoyo fundamental para los hijos gays e hijas lesbianas. Se las suele describir como agentes reconciliadores entre los distintos miembros de las familias y como aquellas personas que suelen posibilitar la apertura del conocimiento y del relacionamiento con la diversidad sexual de los hijos e hijas a otros miembros, especialmente los padres.

Aunque las investigaciones revisadas evidencian que el apoyo social familiar es un aspecto relacionado con efectos positivos en la vida de hijos e hijas homosexuales, también es notorio que este tipo de apoyo se ha estudiado en gran medida de manera descriptiva y correlacional con otras variables, así como los procesos de revelación y aceptación que se han generado a nivel personal, familiar y social. Por tanto, a nuestro juicio, se requiere incursionar en otros tipos de acercamiento investigativo sobre el tema. Especialmente en el contexto colombiano es necesario poder abordar el apoyo social percibido y recibido desde las experiencias de padres, madres, hijos gays e hijas lesbianas, así como el apoyo informacional, material y — de manera fundamental- emocional. De igual manera, es indispensable poder conocer las experiencias que vivencian los papás en estas familias, dado que en las investigaciones revisadas gran parte de las participantes eran madres. Igualmente importante es poder continuar reconociendo estas experiencias de familia, teniendo también en cuenta otras variables como son el nivel socioeconómico y educativo, la edad cronológica de los hijos e hijas, la identidad étnica, la procedencia rural o urbana, la zona del país, etc., pues se entiende que las familias son diversas en muchos aspectos.

Otras dimensiones de análisis no cubiertas en los estudios revisados podrían incluir el reconocimiento de los efectos positivos del apoyo social familiar en familias con hijos gays y lesbianas; por ejemplo, el de los padres y madres como pareja o como figuras parentales dentro o fuera de una relación particular, así como el impacto en las relaciones fraternales y con el subsistema familiar con las redes familiares extensas o con otras redes como la escolar, la religiosa, entre otras.

En suma, se puede señalar que un entendimiento complejo de las experiencias de revelación, manejo y aceptación de la orientación sexual de los hijos gays e hijas lesbianas en las familias deberá trascender las relaciones que implican directamente al hijo o hija para identificar y acompañar las movilizaciones que se generan entre las otras redes y subsistemas. Desde la investigación se hace necesario comprender más de manera situada, teniendo en cuenta una red amplia de personas/relaciones y los tipos de apoyo que más favo- 
recen el bienestar físico, psicológico y social en las personas, especialmente en aquellas con orientaciones sexuales diversas.

Por último, y como se ha venido argumentando, se recomienda para futuras investigaciones el reconocer al apoyo social — particularmente el familiar- como un proceso transversal, presente y activo en las relaciones familiares. Se hace necesario y urgente continuar estudiando y documentando las experiencias de diferentes miembros de la familia tanto nuclear como extensa, de forma tal que se expanda y profundice el conocimiento de las familias con hijos gays e hijas lesbianas que, desde sus propias narrativas, brindan dicho apoyo social familiar.

\section{Agradecimientos}

Los autores agradecemos el apoyo financiero por parte de la Oficina de Investigación y Desarrollo de la Pontificia Universidad Javeriana Cali al proyecto de investigación realizado por el grupo de investigación Bienestar, trabajo, cultura y sociedad (Bitacus), en el año 2018, denominado «Dinámicas familiares en el proceso de revelación y aceptación de la orientación sexual de hijos gays e hijas lesbianas en Cali, Colombia» (código: 020100594), del cual se deriva el presente artículo. Además, expresamos nuestra gratitud a las familias que participaron compartiendo su historia para hacer posible este estudio, a los y las integrantes del semillero de investigación Conversex: conversemos sobre sexualidad de la misma universidad, al monitor del proyecto de investigación y estudiante de psicología Juan Sebastian Rueda-Toro y a la Psicóloga Marcela Tinoco por su apoyo en la realización, transcripción, codificación y análisis de las entrevistas.

\section{Referencias}

Las referencias marcadas con asterisco indican estudios incluidos en la revisión.

*Apoorva, B., \& Thomas, E. (2016). Interpersonal experiences of lesbians with their parents: An interpretative phenomenological analysis. Journal of Psychosocial Research, 11(2), 251-258.

*Aranda, F., Matthews, A. K., Hughes, T. L., Muramatsu, N., Wilsnack, S. C., Johnson, T. P., \& Riley, B. B. (2015). Coming out in color: Racial/ethnic differences in the rela- 
tionship between level of sexual identity disclosure and depression among lesbians. Cultural Diversity and Ethnic Minority Psychology, 21(2), 247-257. https://doi.org/ 10.1037/a0037644

*Baiocco, R., Fontanesi, L., Santamaria, F., Ioverno, S., Marasco, B., Baumgartner, E., Willoughby, B., \& Laghi, F. (2015). Negative parental responses to coming out and family functioning in a sample of lesbian and gay young adults. Journal of Child and Family Studies, (24), 1490-150o. https://doi.org/10.1007/s10826-014-9954-z

*Baptist, J. A., \& Allen, K. R. (2008). A family's coming out process: Systemic change and multiple realities. Contemporary Family Therapy, (30), 92-110. https://doi.org/ 10.1007/s10591-008-9057-3

*Bautista, E. (2018). Reflexiones acerca de la diversidad sexual entre jóvenes indígenas en México. Revista de Estudios Sociales, (63), 100-109. https://doi.org/10.7440/ res63.2018.08

*Biswas, D., \& Chaudhuri, A. (2018). Perks of coming out of the closet: From the perspective of LGBTQ individuals. Indian Journal of Health and Wellbeing, 9(2), 239-246.

*Bond, B. J., Hefner, V., \& Drogos, K. L. (2009). Information-seeking practices during the sexual development of lesbian, gay, and bisexual individuals: The influence and effects of coming out in a mediated environment. Sexuality \& Culture: An Interdisciplinary Quarterly, 13(1), 32-50. https://doi.org/10.1007/s12119-008-9041-y

Cass, V. (1979). Homosexuality identity formation: A theoretical model. Journal of Homosexuality, 4(3), 219-235. https://doi.org/10.1300/Jo82v04no3_01

*Cassar, J., \& Grima, M. (2016). Sex is a minor thing: Parents of gay sons negotiating the social influences of coming out. Sexuality \& Culture, (20), 987-1002. https://doi.org/ 10.1007/s12119-016-9368-8

*Ceballos-Fernández, M. (2014). Identidad homosexual y contexto familiar heteroparental: implicaciones educativas para la subversión social. Revista Latinoamericana de Ciencias Sociales, Niñez y Juventud, 12(2), 643-658. https://doi.org/10.1160o/1692715x.1229140514

*Chan, K. K. (2017). Family and homosexuality in Chinese culture: Rights claims by non-heterosexuals in Hong Kong. Sexuality \& Culture, (21), 845-859. https://doi.org/ $10.1007 /$ s12119-017-9425-y

Colombia Diversa \& Sentiido. (2016). Mi voz cuenta: encuesta de clima escolar LGBT en Colombia 2016. https://bit.ly/3g2Bgba

Espinal, I., Gimeno, A., \& González, F. (2006). El enfoque sistémico en los estudios sobre la familia. Revista Internacional de Sistemas, (14), 21-34. 
Feeney, B., \& Collins, N. L. (2015). A new look at social support: A theoretical perspective on thriving through relationships. Personality and Social Psychology Review, 19(2), 1-35. https://doi.org/10.1177/1088868314544222

*Figueroa, V., \& Tasker, F. (2013). «I Always have the idea of sin in my mind....»: Family of origin, religion, and chilean young gay men. Journal of GLBT Family Studys, 10(3), 269-297. https://doi.org/10.1080/1550428X.2013.834424

*Flentje, A., Bacca, C., \& Cochran, B. (2015). Missing data in substance abuse research? Researchers' reporting practices of sexual orientation and gender identity. Drug and Alcohol Dependence, 147 (1), 280-284. https://doi.org/10.1016/j.drugalcdep.2014.11.012

*González, K., Rostosky, S. S. Odom, R. D., \& Riggle, E. D. B. (2013). The positive aspects of being the parent of an LGBTQ Child. Family Process, 52(2), 325-337. https:// doi.org/10.1111/famp.12009

*Goodrich, K. M. (2009). Mom and dad come out: The process of identifying as a heterosexual parent with a lesbian, gay, or bisexual child. Journal of LGBT Issues in Counseling, 3(1), 377-61. https://doi.org/10.1080/15538600902754478

*Guittar, N. A., \& Rayburn, R. L. (2016). Coming out: The career management of one's sexuality. Sexuality \& Culture, (20), 336-357. https://doi.org/10.1007/s12119-015-9325-y

*Hatzenbuehler, M. L. (2011). The social environment and suicide attempts in lesbian, gay, and bisexual youth. Pediatrics, 127(5), 896-903. https://doi.org/10.1542/ peds. $2010-3020$

Hernández, A. (2001). El enfoque sistémico como fundamento epistemológico de la intervención con familias. En Familia e Intervención sistémica breve en diversos contextos (pp. 9-22). Pontificia Universidad Javeriana.

Hernández, Y. (2006). Acerca del género como categoría analítica. Nómadas Revista Crítica de Ciencias Sociales y Jurídicas, 13(1), 1-10.

*Jiménez, A., \& Romero, M. (2014). «Salir del clóset» en la ciudad de México. Salud Mental, $37(5), 391-397$.

*Kabakov, M. (2014). The parents are our future: Orthodox communities' openness to their LGBT members. Journal of Jewish Communal Servive, 89(1), 61-67.

*Lagazzi, I. M. (2016). Construcción subjetiva de adolescentes y jóvenes lesbianas y gays acerca del rol de la familia en sus procesos suicidas [Tesis de maestría, Universidad Alberto Hurtado]. Repositorio Universidad Alberto Hurtado. https://bit.ly/2XhFpeh

*Luján, I., \& Tamarit, A. (2012). Dinámica familiar ante la revelación de la orientación homosexual de los hijos/as. International Journal of Developmental and Educational Psychology, 3(1), 301-308. 
*Manning, J. (2015). Communicating sexual identities: A typology of coming out. Sexuality \& Culture, (19), 122-138. https://doi.org/10.1007/s12119-014-9251-4

*McConnell, E. A., Birkett, M., \& Mustanski, B. (2016). Families matter: Social support and mental health trajectories among lesbian, gay, bisexual, and transgender youth. Journal of Adolescent Health, 59(6), 1-7. http://dx.doi.org/10.1016/j.jadohealth.2016.07.026

Ministerio de Salud \& Profamilia. (2015). Encuesta Nacional de Demografía y Salud 2015. Tomo I. https://bit.ly/2WPgb7U

*Montenegro, J. L., Orcasita, L. T., Guayara, L. A., \& Zapata, L. J. (2019). Representaciones sociales sobre masculinidad y paternidad en padres con hijos gays. Revista de Investigación en Enfermería: Imagen y Desarrollo, 21(1). https://doi.org/10.11144/ Javeriana.ie21-1.rsmp

*Mustanski, B., Newcomb, M. E., \& Garofalo, R. (2011). Mental health of lesbian, gay, and bisexual youths: A developmental resiliency perspective. Journal of Gay \& Lesbian Social Services, 23(2), 204-225. https://doi.org/10.1080/10538720.2011.561474

Núñez, G. (2016). ¿Qué es la diversidad sexual? Ariel.

*Ocampo, A. C. (2014). The gay second generation: Sexual identity and family relations of filipino and latino gay men. Journal of Ethnic and Migration Studies, 4O(1), 155-173. https://doi.org/10.1080/1369183X.2013.849567

*Orcasita, L. T., Sevilla, T. M., Valencia, C., \& Palma, D. (2015, septiembre). Dinámicas familiares y abordaje de la sexualidad en jóvenes universitarios que se autodenominan homosexuales [Ponencia]. Congreso Colombiano de Psicología «1ra Conferencia Regional Latinoamericana de Psicología», Armenia, Colombia.

Orcasita, L. T., \& Uribe, A. F. (2010). La importancia del apoyo social en el bienestar de los adolescentes. Psychologia: Avances de la Disciplina, 4(2), 69-82.

Pérez, B. (2005). Homosexualidad: secreto de familia: el manejo del secreto en familias con algún miembro homosexual. Eagles.

*Perrin-Wallqvist, R., \& Lindblom, J. (2015). Coming out as gay: A phenomenological study about adolescents disclosing their homosexuality to their parents. Social Behaviour and Personality, 43(3), 467-80. http://dx.doi.org/10.2224/sbp.2015.43.3.467

*Phillips, M. J., \& Ancis, J. R. (2008). The process of identity development as the parent of a lesbian or gay male. Journal of LGBT Issues in Counseling, 2(2), 126-158. https:// doi.org/10.1080/15538600802125605

Pinos, V. P., Pinos, G. M., \& Palacios, M. D. (2011). Percepciones sobre diversidad sexual en adolescentes escolarizados de la ciudad de Cuenca. Maskana, 2(2), 39-55. https:// doi.org/10.18537/mskn.02.02.04 
*Pistella, J., Salvati, M., Ioverno, S., Laghi, F., \& Baiocco, R. (2016). Coming-out to family members and internalized sexual stigma in bisexual, lesbian and gay people. Journal of Child and Families Studies, (25), 3694-3701. https://doi.org/10.1007/ S10826-016-0528-0

*Plöderl, M., Wagenmakers, E. J., Tremblay, P., Ramsay, R., Kralovec, K., Fartacek, C., \& Fartacek, R. (2013). Suicide risk and sexual orientation: A critical review. Archives of Sexual Behavior, 42(5), 715-727. https://doi.org/10.1007/s10508-012-0056-y

*Quintanilla, R., Sánchez-Loyo, L. M., Correa-Márquez, P., \& Luna-Flores, F. (2015). Proceso de aceptación de la homosexualidad y la homofobia asociados a la conducta suicida en varones homosexuales. Masculinities \& Social Change, 4(1), 1-25. https:// doi.org/10.4471/MCS.2015.58

*Ronzón-Tirado, R. C., Yedra, L. R. y González-Flores, M. P. (2017). Modelos parentales y su relación con la violencia en las parejas del mismo sexo. Revista Latinoamericana de Ciencias Sociales, Niñez y Juventud, 15(2), 1137-1147. https://doi.org/ 10.11600/1692715x.1522317062016

*Rueda, M. A. (2015). El significado del prejuicio sexual en las trayectorias de la identidad con la orientación sexual homosexual y bisexual [Tesis doctoral no publicada]. Universidad de los Andes.

*Ryan, W. S., Legate, N., \& Weinstein, N. (2015). Coming out as lesbian, gay, or bisexual: The lasting impact of initial disclosure experiences. Self and Identity, 14 (5), 549-569. http://dx.doi.org/10.1080/15298868.2015.1029516

*Saltzburg, S. (2007). Narrative therapy pathways for re-authoring with parents of adolescents coming-out as lesbian, gay, and bisexual. Contemporary Family Therapy, 29, 57-69. https://doi.org/10.1007/s10591-007-9035-1

Savin-Williams, R. C., \& Dubé, E. (1998). Parental reactions to their child's disclosure of a gay/lesbian identity. National Council on Family Relations, 47(1), 7-13. https:// doi.org/10.2307/584845

*Snapp, S. D., Watson, R. J., Russell, S. T., Díaz, R. M., \& Ryan, C. (2015). Social support networks for LGBT young adults: Low cost strategies for positive adjustment. Family Relations, 64(3), 420-430. https://doi.org/10.1111/fare.12124

*Solís, F. (2014). Proceso de aceptación que experimentan padres y madres de hijos homosexuales ante el conocimiento de la orientación sexual. Revista Científica de FAREM-Esteli, (12), 28-41. http://dx.doi.org/10.5377/farem.v3i12.2459 
*Solomon, D., McAbee, J., \& McGee, A. (2015). Coming out and the potential for growth in sexual minorities: The role of social reactions and internalized homonegativity. Journal of Homosexuality, 62(11), 1512-1538. https://doi.org/10.1080/00918369.2015.1073032

*Svab, A. (2016). Narratives of coming out to parents: Results of replicating a sociological study on the everyday life of gays and lesbians in slovenia (2014-2015). Teorija in praksa, 53(6), 1344-1355.

Szasz, I. (2004). El discurso de las ciencias sociales sobre sexualidades. En C. Cáceres (Ed.), Ciudadanía sexual en América Latina: abriendo el debate (pp. 65-75). Universidad Peruana Cayetano Heredia.

*Tamagawa, M. (2018). Coming out to parents in Japan: A sociocultural analysis of lived experiences. Sexuality \& Culture, 22(2), 497-520. https://doi.org/10.1007/ S12119-017-9481-3

*Trussell, D. E., Xing, T. M. K., \& Oswald, A. G. (2015). Family leisure and the coming out process for LGB young people and their parents. Annals of Leisure Research, 18(3), 323-341. http://dx.doi.org/10.1080/11745398.2015.1075224

*Tyler, T. R. (2015). Our story: The parent and LGBTQ child relational process. Journal of Gay \& Lesbian Social Services, 27(1), 17-45. https://doi.org/ $10.1080 / 10538720.2015 .988313$

*Uribe, M., Javier, E. S., \& Artotoma, R. M. (2018). Actitudes de los padres hacia la homo-sexualidad de sus hijos. Horizonte de la Ciencia, 8(15), 71-81.

*Vargas, E., Ripoll, K., Carrillo, S., Rueda, M., \& Castro, J. A. (2011). Experiencias familiares de madres y padres con orientaciones sexuales diversas: aportes desde la investigación. Universidad de los Andes. https://doi.org/10.7440/2013.49

Walsh, F. (2016). Strengthening family resilience. Guilford Press.

*Zambrano, C., Ceballos, A., \& Ojeada, D. (2017). Reconocimiento de la orientación homosexual. Revista Psicoespacios, 11(19), 77-93.

*Zambrano, C., Hernández, P., \& Guerrero, A. (2019). Proceso de reconocimiento de la orientación sexual homosexual en estudiantes de una universidad pública. Psicogente, 22(41), 1-29. https://doi.org/10.17081/psico.22.41.3310 